\title{
Effects of Time Delay in Cardiac Blood Flow Measurements by Bolus $\mathrm{H}_{2}{ }^{15} \mathrm{O}$
}

\author{
Sinisa Pajevic, Member, IEEE, Stephen L. Bacharach,* Member, IEEE, \\ Richard E. Carson, Member, IEEE, and George H. Weiss
}

\begin{abstract}
Myocardial blood flow (rMBF) can be measured using dynamic positron emission tomography (PET) and bolus injection of $\mathrm{H}_{2}{ }^{15} \mathrm{O}$. Recent studies indicate that large errors in the estimates of flow $(\hat{f})$ can be produced by time shifts between the true arterial input function and the measured input function $[A(t)]$. We have investigated this phenomenon further using $A(t)$ derived from patient data, and using simulated myocardial time activity curves $[M(t)]$. We found that with judicious choice of scan parameters and region of interest (ROI) placement, these errors can be greatly reduced. In particular, when $A(t)$ is measured from the left ventricular (LV) cavity, the bias in $\hat{f}$ is negligible over a wide range of circumstances. However, when $A(t)$ is not measured from the $\mathbf{L V}$ cavity, the bias in flow can be large for short scans $(<2$ min) or low flow values $(f<0.4$ $\mathrm{ml} / \mathrm{g} / \mathrm{min})$. We show that the bias is primarily due to the spillover term in the model that is most commonly used to compute rMBF and suggest some correction methods. We conclude that it is possible to avoid errors in estimates of flow due to time delay.
\end{abstract}

Index Terms - Blood flow, cardiac imaging, Kety model, positron emission tomography.

\section{INTRODUCTION}

$\mathbf{P}$ OSITRON emission tomography (PET) permits the concentration of radiotracers in small regions of tissue to be measured as a function of time. Such dynamic measurements can be used to compute regional myocardial blood flow (rMBF), a parameter of great clinical importance. One of the most accurate ways to measure $\mathrm{rMBF}$ is by measuring the arrival and/or clearance of ${ }^{15} \mathrm{O}$ water from the myocardium. Several methods have been used to analyze such data. Some of these methods require a slow delivery of the tracer to the myocardial tissue, either by slow infusion of $\mathrm{H}_{2}^{15} \mathrm{O}$ or by inhalation of $\mathrm{C}^{15} \mathrm{O}_{2}$ [1], [2]. Another widely used method requires a rapid delivery of $\mathrm{H}_{2}^{15} \mathrm{O}$ to the myocardium, by bolus injection [2]-[10]. While this bolus method has certain advantages[10] it has recently been shown to be quite sensitive to time delays between the measured arterial curve $[A(t)]$ and

Manuscript received February 20, 1996; revised March 11, 1997. The Associate Editor responsible for coordinating the review of this paper and recommending its publication was D. Townsend. Asterisk indicates corresponding author.

S. Pajevic and G. H. Weiss are with the Physical Sciences Laboratory, Division of Computer Research and Technology, National Institutes of Health, Bethesda, MD 20982 USA.

*S. L. Bacharach is with the Nuclear Medicine Department, Clinical Center, National Institutes of Health, Bldg. 10, rm. 1C401, Bethesda, MD 20982 USA (e-mail: steve@nmdhst.cc.nih.gov).

R. E. Carson is with the PET Department, Clinical Center, National Institutes of Health, Bethesda, MD 20982 USA.

Publisher Item Identifier S 0278-0062(97)04237-7.

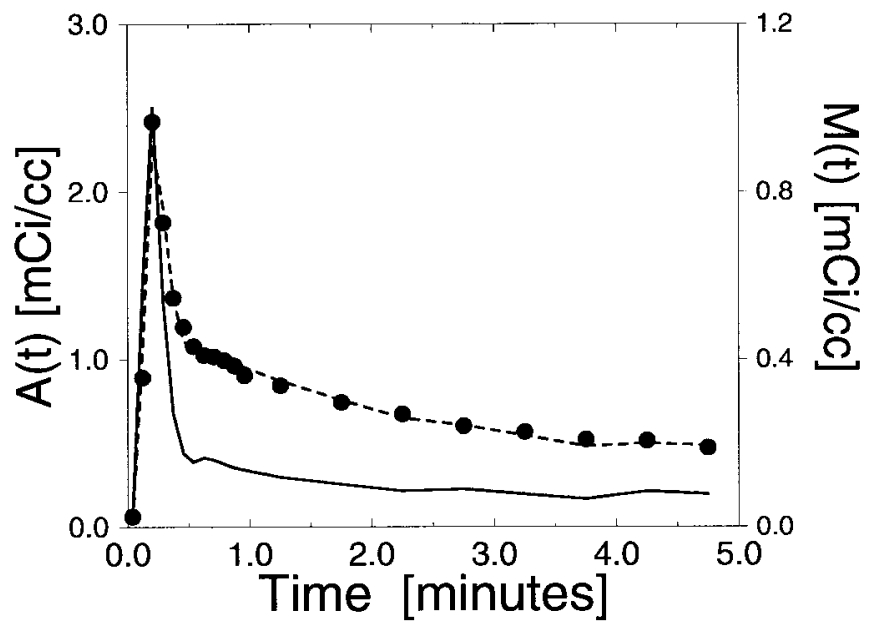

Fig. 1. An arterial input curve, $A(t)$, (solid line), and a myocardial tissue TAC, $M(t)$, (solid circles) measured in left-wall. The dashed line represents fit of $M(t)$ to the model in (1) with the parameter estimates $\hat{f}=0.94$ $\mathrm{ml} / \mathrm{g} / \mathrm{min}, S=0.32$, and $P=0.72$. When using a four parameter model $\left(C_{\mathrm{SC}}\right.$, see Sections III-B and IV-A for details) we obtain $\hat{f}=0.92 \mathrm{ml} / \mathrm{g} / \mathrm{min}$, $S=0.33$, and $P=0.71$ and $\Delta t_{2}=1 \mathrm{~s}$.

the arrival of activity in the tissue [11]. The purpose of this paper is to re-examine this sensitivity, and to suggest several ways in which the advantages of the bolus method can be retained, while removing the sensitivity of the method to small time delays.

In the standard bolus model, the myocardial time activity curve, $M(t)$, is fitted to a function which represents Kety's single-compartment model [12], [13], with an added spill-over term. This term accounts for the "spill-over" of counts [3]-[7] from the left ventricular (LV) cavity into the myocardium. This model contains three parameters in addition to the flow rate $f$. These are the partial volume correction factor $(P)$, the spillover factor $(S)$, and the tissue/blood partition coefficient $(\lambda)$, generally assumed to have a constant value equal to $0.91 \mathrm{ml} / \mathrm{g}$. This modified Kety equation, is expressed in terms of $A(t)$ as

$$
M(t)=P\left[f e^{-f t / \lambda} \otimes A(t)\right]+S \cdot A(t)
$$

where $\otimes$ designates a convolution process, and both $A(t)$ and $M(t)$ are corrected for decay. The first term in (1) will be referred to as the convolution term and the second one as the spill-over term.

Time activity curves are derived from the time sequence of PET images by defining appropriate regions of interest (ROI's). ROI's used to estimate $A(t)$ are usually placed in the 
left atrium or left ventricle and those used to estimate $M(t)$, are placed inside the myocardium. Fig. 1 shows a typical arterial input curve (solid line) and a measured myocardial time activity curve (TAC) (solid circles), illustrating the shapes of the two curves. In this case, $A(t)$ was determined from an ROI in the left atrium. A very small time shift may occur between $M(t)$ and $A(t)$. Such time shifts are due to the small, but finite, time required for blood to travel from the point where $A(t)$ is measured to the $\mathrm{LV}$ cavity and to the myocardium. While the time delay is generally very small $(\approx 1 \mathrm{~s})$ it has been reported [10], [11] that its effects on the estimate of flow $(\hat{f})$ can be significant. We wished to examine these findings and postulated that there might be certain circumstances in which these previously reported systematic errors in flow can become very small. By examining the behavior of the model used to measure flow, we determined whether such circumstances do indeed exist, and if so, how to utilize them clinically to produce flow estimates with only a small systematic error. In addition, there may be some cases in which these circumstances cannot be achieved. In this case, we explored the possibility of correcting for the time shift by introducing a fourth parameter (a time delay) in addition to those appearing in (1). Such corrections have been tried previously in the context of measurements of regional cerebral flow (rCBF) [14], but the presence of substantial spillover makes the measurement of cardiac flow a very different problem.

\section{Methods}

In order to address the time delay problem and its influence on the estimate of myocardial blood flow obtained in dynamic PET, we investigated a generalization of Kety's model in which time shifts are introduced into (1). We measured arterial curves from human subjects and used these curves and the generalized Kety model to simulate tissue time-activity curves. Gaussian noise was then added to the resulting curves. The use of simulated, rather than the real, tissue curves enabled us to study the effect of the time shift for different values of relevant parameters like flow, spill-over, and total scanning time $\left(T_{t s}\right)$, while the use of arterial curves from human subjects insured the myocardial curves had a realistic and clinically relevant shape.

\section{A. Kety Equation with Time Delays}

The arterial input functions included in the spill-over and convolution terms may be misaligned. Therefore, we needed to extend (1) to take this effect into account. In order to separate the effects of atrial-LV-myocardial time delays from other potential difficulties associated with the bolus model, we have ignored any influence right ventricular (RV) activity might have (as might occur in a septal region [2]), and have assumed that the spill-over of counts into the myocardium comes primarily from the LV cavity. In this case the time-delay effects can be modeled using two delay times. The first time delay, $\Delta t_{1}$, is the time required for the bolus to move from the point at which $A(t)$ is measured, to the myocardium, and the second time delay, $\Delta t_{2}$, represents the (slightly shorter) time for the bolus to reach the LV cavity from the measurement point. Using these definitions (1) becomes

$$
M(t)=P\left[f e^{-f t / \lambda} \otimes A\left(t-\Delta t_{1}\right)\right]+S \cdot A\left(t-\Delta t_{2}\right) .
$$

In order to examine the importance of each of the terms in (2) and, also, in order to limit the number of free parameters when fitting $M(t)$ to (2), we studied two different cases.

1) Setting $\Delta t_{2}=0$, corresponding to the case in which $A(t)$ is measured from the LV cavity. In what follows this case will be referred to as $\mathbf{C}_{\mathrm{CO}}(\mathrm{CO} \equiv$ Convolution Only-indicating that the time delay is included only in the convolution term).

2) Setting $\Delta t_{1}=\Delta t_{2}+\delta t$, where $\delta t$ represents the time for the bolus to travel from the LV cavity to the myocardium and is assumed to be a constant. Here, time delays are introduced in both the spill-over and convolution term and this case will be referred to as $\mathbf{C}_{\mathrm{SC}}$ (Spill-over and Convolution). The flow values obtained by fitting this equation proved to be insensitive to the precise value chosen for $\delta t$, in the range $0-2 \mathrm{~s}$. The $C_{\mathrm{SC}}$ case can be used with $A(t)$ 's measured at any arbitrary location, in contrast to $\mathrm{C}_{\mathrm{CO}}$.

\section{B. Simulation of the Tissue Time Activity Curves}

We measured arterial input curves from the dynamic PET data taken from two subjects, a volunteer with no known cardiac disease and a patient with coronary artery disease. The measurements on the healthy subject were performed using a POSICAM PET scanner with scan durations equal to $3 \mathrm{~s}$ for the first ten scan points and then $30 \mathrm{~s}$ per scan for the remainder of the measurement. The second subject was scanned with a General Electric Advance scanner in 3D mode (septa out). Scan durations for the GE scanner were set to $5 \mathrm{~s}$ for the first 12 scans, and $30 \mathrm{~s}$ for the remaining eight scans. Attenuation correction was performed using a transmission scan. The arterial input curves were measured from each patient, using an ROI mainly in the left atrium. Tissue activity curves were created from the measured arterial input function using (2). In other words we used the arterial input curves measured in the LA as a template and then, using appropriate time-shifts, we emulated the arterial input curves which would have been measured elsewhere. Measured TAC's from the LA, the LV, and the aorta indicated that dispersion was exceedingly small during the short LA-LV-Aorta transit time-e.g., the full width at half-max of the curves changed by less than $2.0 \%$ for the biggest assumed transit time. The effects of dispersion were, therefore, ignored in our model. Finally, we added Gaussian noise to these curves to create an ensemble of noisy realizations of the given curve. The amount of noise was chosen to accurately mimic noise occuring in PET tissue curves. The PET scanner produced images by correcting the accumulated coincidences $N_{c}$ [equal to the sum of the true $(T)$ and random $(R)$ coincidences, i.e., $\left.N_{c}=T+R\right]$ by the factor $\left(F_{d t} F_{d c} / T_{s}\right)$, where $F_{d t}$ (dead time correction factors), $F_{d c}$ (decay correction factor), $T_{s}$ (scan durations), and $N_{c}$ are derived from actual scan data. Therefore, the amount of noise added in our simulations was proportional 


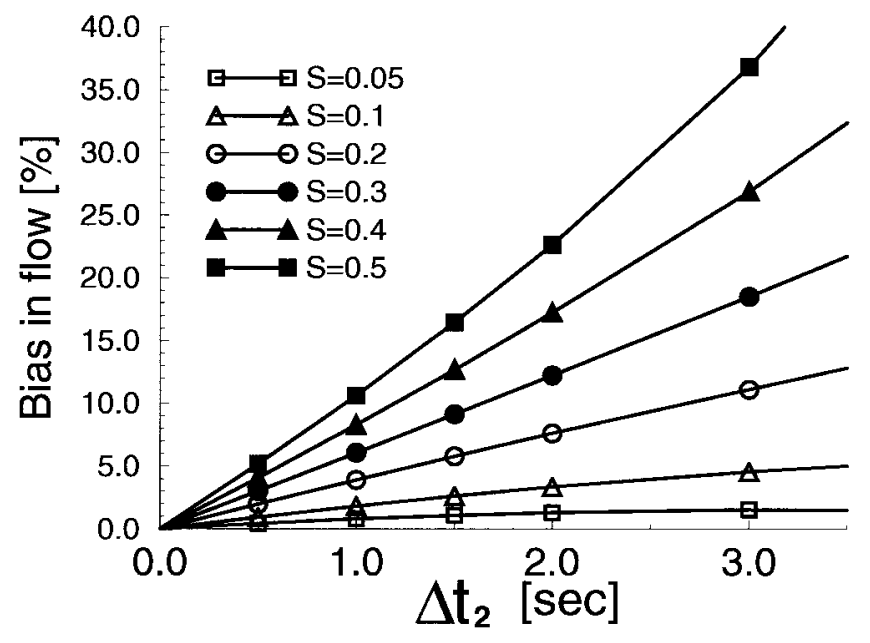

Fig. 2. Flow bias as a function of time delay modeled according to $\mathrm{C}_{\mathrm{SC}}$ for six different values of spill-over $\left(f=1 \mathrm{ml} / \mathrm{g} / \mathrm{min} ; T_{t s}=4.25 \mathrm{~min}\right.$; $P=0.8)$. The $A(t)$ and weights $(W)$ were obtained on the POSICAM scanner. The time delay $\Delta t_{2}$ was varied and $\delta t$ was fixed to $0.3 \mathrm{~s}$. The fact that the bias vanishes as spill-over decreases indicates that the second term in (2) is the main source of the bias in flow due to time delay.

to $\left(F_{d t} F_{d c} / T_{s}\right) \sqrt{N_{c}}$. When fitting the resulting tissue curves according to $\mathrm{C}_{\mathrm{CO}}$ and $\mathrm{C}_{\mathrm{SC}}$, the fits were weighted with factors $(W)$, which were inversely proportional to the variance, i.e.,

$$
W=\frac{T_{s}^{2}}{F_{d t}^{2} F_{d c}^{2}} \frac{1}{N_{c}} .
$$

A range of noise levels was studied, and the noise level was specified as the percent error $\left(P_{E}\right)$ at the last data point. We also created TAC's of various durations (from 1.25-4.75 min) to determine how the total scanning time $T_{t s}$ influences the relationship between $\hat{f}$ and time delay. Finally, we wished to determine how the introduction of the time delay parameter influenced systematic errors and noise in the estimate of flow, $\hat{f}$. To this end 400 noisy realizations of each myocardial activity curve were produced from which the bias and standard deviation (SD) of $\hat{f}$ could be estimated.

\section{RESULTS}

\section{A. Systematic Errors in $\hat{f}$}

We first investigated the systematic error, or bias, in $\hat{f}$ produced if a time delay in fact exists but is ignored in fitting the data. To determine this error, we created tissue time activity curves with a range of time delays and with a low noise level $\left(P_{E}<1 \%\right)$ which were then fit to (1). We studied the effects of the spill-over value $(S)$, total scanning time $\left(T_{t s}\right)$, and flow value $(f)$ on the bias introduced by a time delay.

1) Spill-Over: We presumed that $S$, the fraction of $\mathrm{LV}$ counts spilling over into the myocardial ROI's, would strongly influence the sensitivity of $\hat{f}$ to time delay. Fig. 2 illustrates the dependence of the bias in $\hat{f}$ on the time delay for six different values of spill-over, and with $\left(f=1 \mathrm{ml} / \mathrm{g} / \mathrm{min} ; T_{t s}=4.25\right.$ $\min ; P=0.8)$. Note that the value of partial volume $(P)$ and spillover $(S)$ must, for physical reasons, sum to a value less than or equal to one. However, in the simulations of Fig. 2 (and elsewhere) values of $P$ and $S$ are used which often do

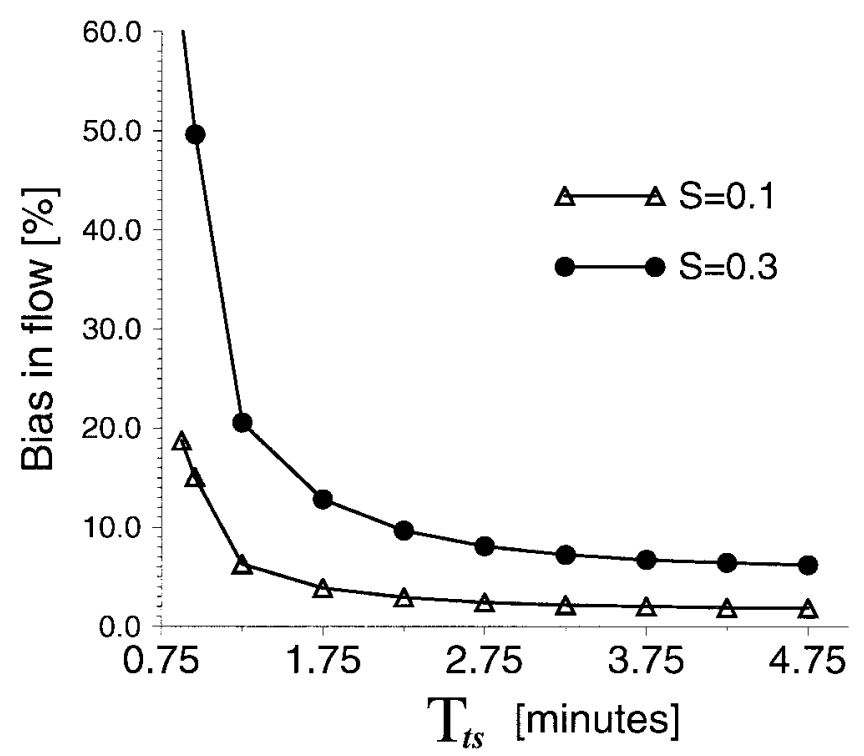

Fig. 3. Flow bias as a function of the total scanning time $\left(T_{t s}\right)$ for two spill-over values and parameters $\Delta t_{1}=1.3 \mathrm{~s} ; \Delta t_{2}=1 \mathrm{~s} ; f=1 \mathrm{ml} / \mathrm{g} / \mathrm{min}$ and $P=0.8 . A(t)$ and weights $(W)$ were obtained on the GE scanner. Similar results were obtained with POSICAM derived weights. This figure shows, clearly, that the total scanning time has a great influence on the sensitivity of the measured flow to the time discrepancy between $A(t)$ and $M(t)$.

not sum to less than unity. This was done for simplicity, so that the figures could show the effect of varying only a single parameter for wide ranges of that parameter. As can be seen from (1) and (2), requiring $S+P \leq 1$ is a physical, not a mathematical, constraint. It is the relative value of $S$ and $P$ which matters, not their absolute values. Therefore, the values of $S$ and $P$ in Fig. 2 (and elsewhere) may be scaled by any factor, as long as the $S / P$ value remains unchanged. The range of spill-over values used in the simulation (0.1-0.5) included the range of values typically found in clinical practice $(0.2-0.4)$. In Fig. 2, $\mathrm{C}_{\mathrm{SC}}$ was used as the model. The time delay $\Delta t_{2}$ was varied and $\delta t$ was fixed at $0.3 \mathrm{~s}$. This figure indicates that the systematic error increases monotonically with time delay and that the rate of the increase depends strongly on the value of $S$. For low values of spill-over, bias in $\hat{f}$ is negligible for all time delays, while for large spill-over, bias in $\hat{f}$ can become large. Thus the spill-over term in (2) has a large effect on the systematic error produced by neglecting time delay. However, even at large values of $S$, a 1-s delay produces less than $10 \%$ bias in flow.

2) Total Scanning Time: Since the spill-over term becomes small soon after injection, it is reasonable to suppose that the duration of the measurement, i.e., total scanning time $T_{t s}$, might influence the bias. In other words, by collecting enough data at later times, when the influence of $S$ is insignificant, the sensitivity of flow to time delay might be reduced. Fig. 3 shows the dependence of the bias in flow on $T_{t s}$ for two different spill-over values, using the $\mathrm{C}_{\mathrm{SC}}$ model to simulate data, with the parameter values $\delta t=0.3 \mathrm{~s} ; \Delta t_{2}=1 \mathrm{~s} ; f=1$ $\mathrm{ml} / \mathrm{g} / \mathrm{min} ; P=0.8$. The systematic error in $\hat{f}$ becomes quite large when short scan times are used. It should be pointed out that short scan times-as short as 1.25 min-have often been used when performing bolus water studies [11]. 
3) Weighting Factors: The beneficial effects of increasing scan time are accentuated by the weighting factors used when fitting the data to the model. Since deadtime and randoms are high during the early times, the variance of data collected during these times is also high. Later time points (e.g., from $1.25-5 \mathrm{~min}$ ) typically have lower variance. Therefore, these later time points are weighted more heavily than data from early times, and adding them further reduces the influence of the early, spill-over dominated, time points. Use of appropriate weighting, therefore, is very important. For example, in the extreme case in which no weighting is used we found that the systematic error in $\hat{f}$ for a typical situation $\left(\Delta t_{1}=1\right.$ $\mathrm{s}, \Delta t_{2}=0.7 \mathrm{~s}, T_{t s}=4.25 \mathrm{~min}, f=1 \mathrm{ml} / \mathrm{g} / \mathrm{min}$, and $S=0.3)$ was close to $23 \%$ as compared to only $4 \%$, obtained when proper weights (computed from our POSICAM scanner) were used. Scanners with different deadtime and randoms characteristics require different weighting factors to be used. In order to investigate the influence of different weighting factors we changed the assumed noise characteristics of our data, assuming that a hypothetical "ideal" scanner would have no dead time $\left(F_{d t}=1\right)$, and no random counts $(R=0)$. We then created sets of weights [using (3)] and curves for the ideal scanner, and measured the resulting systematic error. The real scanner (again the POSICAM) produced smaller bias in flow than did the "ideal" scanner $(4.2 \%$ versus $6.4 \%$, for $f=1 \mathrm{ml} / \mathrm{g} / \mathrm{min} ; S=0.3 ; T_{t s}=4.25 \mathrm{~min} ; \Delta t_{1}=1$ $\left.\mathrm{s} \Delta t_{2}=0.7 \mathrm{~s}\right)$. A similar increase in bias was observed for many other combinations of $f, S, T_{t s}$, and $\Delta t$ 's. That is, the flow estimates made using the ideal scanner were more sensitive to time delay than were flows estimated with the real scanner, presumably because the real scanner, with its imperfect randoms and deadtime characteristics, weighted early time points less heavily than an "ideal" scanner.

4) Spill-Over Versus Convolution Term: To investigate further the role of spill-over we compared the bias obtained in $\mathrm{C}_{\mathrm{SC}}$ and $\mathrm{C}_{\mathrm{CO}}$. Fig. 4 shows that the systematic error for short scans in $\mathrm{C}_{\mathrm{CO}}$ (open circles), in which the time delay appears only in the convolution term, is considerably smaller than in $\mathrm{C}_{\mathrm{SC}}$ (solid circles), in which a time delay is also included in the spill-over term. This finding also holds for scans with the more usual 4-5 min duration. For example, for $T_{t s}=4.25$ min, $f=1 \mathrm{ml} / \mathrm{g} / \mathrm{min}, P=0.8$, and $S=0.3$, the bias in $\mathrm{C}_{\mathrm{CO}}$ is $0.13 \%$ for $\Delta t_{1}=1 \mathrm{~s}$, and $4.2 \%$ in $\mathrm{C}_{\mathrm{SC}}$ with $\Delta t_{1}=1 \mathrm{~s}$ and $\delta t=0.3 \mathrm{~s}$. Therefore, time delay has very little effect on flow when $A(t)$ is measured in the $\mathrm{LV}$, but can have a large effect when $A(t)$ is measured elsewhere.

5) Flow Value: We also investigated how the bias in flow, produced by not correcting for time delay, behaves for different flow values. Fig. 5 shows the dependence of the bias on flow in $\mathrm{C}_{\mathrm{CO}}$ and $\mathrm{C}_{\mathrm{SC}}\left(\Delta t_{1}=1 \mathrm{~s}, \delta t=0.3 \mathrm{~s}, T_{t s}=4.25 \mathrm{~min}\right.$, $S=0.3$, and $P=0.8$ ). It shows that in $\mathrm{C}_{\mathrm{CO}}$ the bias remains smaller than $1 \%$ at all physiologically reasonable values of $f$. In $\mathrm{C}_{\mathrm{SC}}$ we see that for flow values $f>0.4 \mathrm{ml} / \mathrm{g} / \mathrm{min}$ the bias is relatively low $(<6 \%)$. However, for very low flows (ischemic regions), the bias in $\hat{f}$ can become much larger. This effect is even greater for scanners with low randoms and dead time (the dashed line in Fig. 5 corresponds to an ideal scanner, $R=0, F_{D T}=1$ ). If, in addition to low flow values,

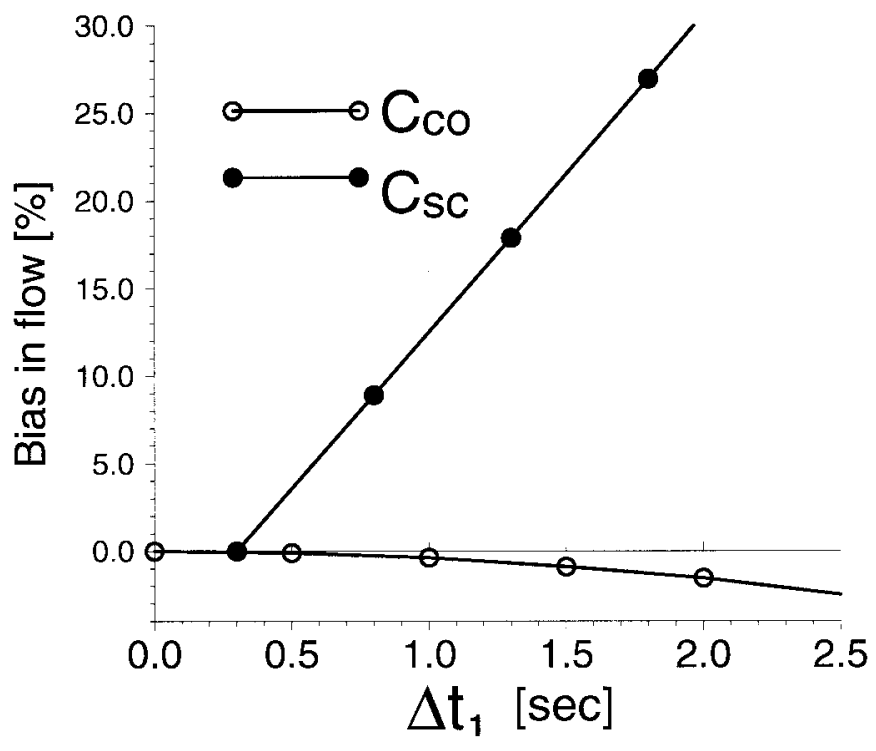

Fig. 4. The absolute value of the flow bias obtained in $\mathrm{C}_{\mathrm{CO}}$ (the bias in $\mathrm{C}_{\mathrm{CO}}$ is negative) is compared to the bias in $\mathrm{C}_{\mathrm{SC}}$ for $T_{t s}=1.25 \mathrm{~min}, f=1$ $\mathrm{ml} / \mathrm{g} / \mathrm{min}, P=0.8$, and $S=0.3$. The weights and the $A(t)$ 's were obtained on the POSICAM scanner. This shows that a time shift in the convolution term produces insignificant error, i.e., a time discrepancy between $M(t)$ and $A(t)$ will produce a significant systematic error only through the spill-over term.

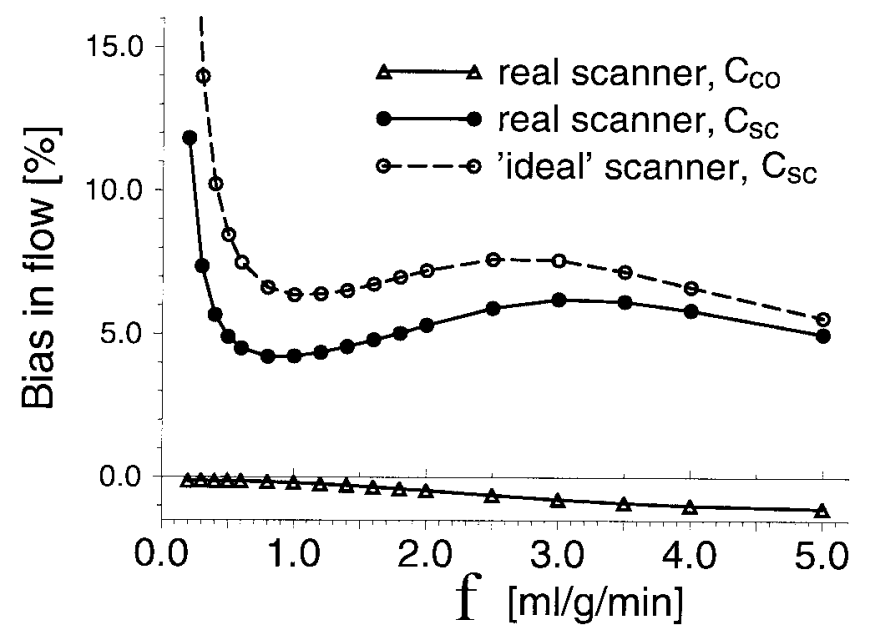

Fig. 5. The dependence of flow bias for $\mathrm{C}_{\mathrm{CO}}$ and $\mathrm{C}_{\mathrm{SC}}$, for real scanner (solid lines) and the ideal scanner (dashed line). The parameters used here are $S=0.3 ; P=0.8 ; T_{t s}=4.25 \mathrm{~min} ; \Delta t_{1}=1 \mathrm{~s}\left(\Delta t_{2}=0.7 \mathrm{~s}\right.$ for $\mathrm{C}_{\mathrm{SC}}$ and zero for $\mathrm{C}_{\mathrm{CO}}$ ). The arterial input curves and weights were obtained on the POSICAM scanner.

the total scanning time is reduced, the systematic error can become extremely large. For example for $f=0.2 \mathrm{ml} / \mathrm{g} / \mathrm{min}$ ( $\Delta t_{1}=1 \mathrm{~s}$ and $S=0.3$ ), the systematic error in $\hat{f}$ increases from $12 \%$ for $T_{t s}=4.25 \mathrm{~min}$ to $70 \%$ for $T_{t s}=1.75 \mathrm{~min}$.

\section{B. Fitting for Time-Delay ( $S D$ in $\hat{f})$}

Because the bias in $\hat{f}$ can be large in certain situations, we investigated the benefit of fitting the data to a four-parameter model using both $\mathrm{C}_{\mathrm{CO}}$ and $\mathrm{C}_{\mathrm{SC}}$ [15]. We examined how well these extensions to (1) reduced the bias in $\hat{f}$, and whether they did so without excessively increasing the SD of $\hat{f}$. We generated 400 noisy replicates of the $M(t)$ using (2). For $\mathrm{C}_{\mathrm{SC}}$, we used $\Delta t_{1}=1.0 \mathrm{~s}$ and $\Delta t_{2}=0.7 \mathrm{~s}$ in (2), and 
TABLE I

The Average Values and the SD (Expressed as the Percent of the Flow Value $f$ ) of $\hat{f}$ in C SC $_{\text {Sc }}$ Obtained From 400 Fits, for Two Different Arterial Input Curves [(a) GE and (b) Posicam Scanners] AND for Several Flow Values. The Introduction of the Fourth Fitting Parameter Does not Change the SD Significantly $\left(\hat{f}_{4}\right)$, Relative to the Three-Parameter Fits $\hat{f}_{3}$ (Fit to Data with no Time Delay) and $\hat{f}_{3}$ Td (Fit to Data with the Time Delay). The Time Delays Used [See (2)] Were $\Delta t_{1}=1 \mathrm{~s}$ and $\Delta t_{2}=0.7 \mathrm{~s}$, Except in $\hat{f}_{3}$ Where Both Times Were Set to Zero. The Other Parameters Were $S=0.3$, $T_{t s}=4.25 \mathrm{~min}, P=0.8$, AND $P_{E}=\{10 \%$ FOR GE SCANNER, 15\% For Posicam SCANNER $\}$

\begin{tabular}{cccc}
\hline $\begin{array}{c}f \\
{[\mathrm{ml} / \mathrm{g} / \mathrm{min}]}\end{array}$ & $\begin{array}{c}\hat{f}_{3 \mathrm{TD}} \\
{[\mathrm{ml} / \mathrm{g} / \mathrm{min}]}\end{array}$ & $\begin{array}{c}\hat{f}_{3} \\
{[\mathrm{ml} / \mathrm{g} / \mathrm{min}]}\end{array}$ & $\begin{array}{l}\hat{f}_{4} \\
{[\mathrm{ml} / \mathrm{g} / \mathrm{min}]}\end{array}$ \\
\hline 0.2 & $0.24 \pm 45.4 \%$ & $0.20 \pm 42.6 \%$ & $0.19 \pm 42.9 \%$ \\
0.3 & $0.32 \pm 31.2 \%$ & $0.30 \pm 30.0 \%$ & $0.29 \pm 29.4 \%$ \\
0.5 & $0.53 \pm 17.5 \%$ & $0.50 \pm 17.9 \%$ & $0.50 \pm 18.1 \%$ \\
1.0 & $1.04 \pm 12.1 \%$ & $1.01 \pm 12.1 \%$ & $1.00 \pm 13.1 \%$ \\
2.0 & $2.12 \pm 11.3 \%$ & $2.00 \pm 10.6 \%$ & $1.99 \pm 12.4 \%$ \\
4.0 & $4.23 \pm 10.9 \%$ & $4.03 \pm 11.4 \%$ & $3.92 \pm 13.0 \%$ \\
\hline
\end{tabular}

(a)

\begin{tabular}{cccl}
\hline $\begin{array}{c}f \\
{[\mathrm{ml} / \mathrm{g} / \mathrm{min}]}\end{array}$ & $\begin{array}{c}\hat{f}_{3_{\mathrm{TD}}} \\
{[\mathrm{ml} / \mathrm{g} / \mathrm{min}]}\end{array}$ & $\begin{array}{c}\hat{f}_{3} \\
{[\mathrm{ml} / \mathrm{g} / \mathrm{min}]}\end{array}$ & $\begin{array}{l}\hat{f}_{4} \\
{[\mathrm{ml} / \mathrm{g} / \mathrm{min}]}\end{array}$ \\
\hline 0.2 & $0.24 \pm 58.7 \%$ & $0.21 \pm 55.9 \%$ & $0.20 \pm 56.4 \%$ \\
0.3 & $0.32 \pm 38.9 \%$ & $0.29 \pm 34.6 \%$ & $0.29 \pm 35.8 \%$ \\
0.5 & $0.53 \pm 24.0 \%$ & $0.51 \pm 23.4 \%$ & $0.50 \pm 23.4 \%$ \\
1.0 & $1.06 \pm 15.5 \%$ & $1.01 \pm 14.6 \%$ & $1.00 \pm 15.1 \%$ \\
2.0 & $2.13 \pm 12.5 \%$ & $2.04 \pm 12.7 \%$ & $2.00 \pm 14.7 \%$ \\
4.0 & $4.27 \pm 12.0 \%$ & $4.01 \pm 11.6 \%$ & $3.83 \pm 15.0 \%$ \\
\hline
\end{tabular}

then fitted the resulting curves according to model $\mathrm{C}_{\mathrm{SC}}(\delta t$ set to $0.3 \mathrm{~s}$, and $\Delta t_{2}$ as the fourth parameter). For $\mathrm{C}_{\mathrm{CO}}$ we used $\Delta t_{1}=1.0 \mathrm{~s}$ and $\Delta t_{2}=0 \mathrm{~s}$ and fitted the resulting curves accordingly ( $\Delta t_{2}$ set to zero and $\Delta t_{1}$ as the fourth parameter). Results for $\mathrm{C}_{\mathrm{SC}}$ are shown in Table I for the arterial input curves derived using the POSICAM and GE scanners and for several values of flow. The estimates of flow for the fourparameter models are labeled as $\hat{f}_{4}$. In order to compare these results to a conventional three parameter model, the same curves were fitted according to (1), obtaining the estimate $\hat{f}_{3 \mathrm{TD}}$. Additionally, we created corresponding curve sets with no time delay and fitted them to (1) to obtain $\hat{f}_{3}$. We can see that the introduction of the fourth fitting parameter effectively reduces or removes the bias in $\hat{f}$, with little or no increase in $\mathrm{SD}$ relative to the three-parameter model. These findings held even when an incorrectly chosen $\delta t$ was used in the fitting procedure $(0<\delta t<2$ s).

We found, using the above simulations, that fitting the data to model $\mathrm{C}_{\mathrm{CO}}$, with $\Delta t_{1}$ as a fourth parameter, can significantly increase the SD of $\hat{f}(\approx 100 \%$ increase over the three parameter model for $T_{t s}=4.25 \mathrm{~min}, f=1 \mathrm{ml} / \mathrm{g} / \mathrm{min}$, $P=0.8, S=0.3$, and $\Delta t_{1}=1 \mathrm{~s}$ ). Although this increase can be reduced by using simultaneous equation regression (fitting several regions simultaneously with the common time delay parameter) [15], it is evident that there is no need to utilize the case $\mathrm{C}_{\mathrm{CO}}$ since the bias was always quite small (Fig. 4). For this reason the $\mathrm{C}_{\mathrm{CO}}$ results are not shown in Table I.

\section{DISCUSSION}

Previous studies [11] have shown that there are small but finite time delays between arterial curves measured from the left atrium, the aorta and the left ventricle. At rest these studies showed an average delay of $0.97 \pm 0.67$ s between the left atrium and the ascending aorta input curves $\left(\Delta t_{2}\right)$, and $0.25 \pm 0.34 \mathrm{~s}$ between the LV and the ascending aorta input curves $(\delta t)$. It has also been reported that even time delays this small could produce large biases in flow, under certain circumstances. Fig. 4, however, shows that when the time delay appears only in the convolution term (case $\mathrm{C}_{\mathrm{CO}}$ ), the bias in $\hat{f}$ is quite small ( $<0.9 \%$ for $\Delta t_{1}$ up to $\left.1.5 \mathrm{~s}\right)$. This clearly indicates that time delays in the convolution term do not affect the estimated flow, $\hat{f}$, significantly. It is only when time delays occur in the spill-over term that $\hat{f}$ is affected. This result has an important practical consequence. If one were to measure the arterial input curve from the LV cavity, then by definition there would be no time delay in the spill-over term. In this situation Fig. 4 shows that the bias is small even in the unlikely case of a large time delay from the LV cavity to the myocardium. Unfortunately, an ROI placed in the LV cavity frequently results in an inaccurate measurement of $A(t)$, due to contamination by myocardial counts, especially in a vigorously contracting ventricle. Such effects can be corrected for, however, using the bidirectional spill-over terms between the myocardium and the LV regions [16]. 
When $A(t)$ is measured at locations other than the LV cavity, however, there is a time delay in the spill-over term, and the bias in $\hat{f}$ increases rapidly with increasing time delay (the solid circles in Fig. 4). On the other hand, Figs. 2 and 3 indicate that this error is reduced if the spill-over fraction, $S$, is small, and if total scanning times, $T_{t s}$, are not short. For example, for $S=0.3$ and $\Delta t_{2}=0.7 \mathrm{~s}$, the bias is reduced from 18 to $6 \%$ when going from $T_{t s}=1.25 \mathrm{~min}$ to $T_{t s}=4.25$ min. If in addition, $S$ has a smaller value of 0.1 (although $S$ is usually larger than this), the bias for $T_{t s}=4.25$ is only $1.8 \%$.

Thus, one way to avoid problems associated with the time delay is to design experiments with long enough total scanning time $\left(T_{t s}>2 \mathrm{~min}\right)$ and in which the myocardial ROI's are drawn so as to avoid overlapping excessively into the LV cavity. In fact, our simulations show that even when the arterial input curve is not measured from the LV, the systematic error produced by neglecting the time delay is lower than $6 \%$ for a wide range of the parameters $\left(f>0.4 \mathrm{ml} / \mathrm{g} / \mathrm{min}, T_{t s}>3\right.$ $\left.\min , S<0.3, \Delta t_{1}<1 \mathrm{~s}\right)$. Previous work with an ammonia flow model [17] has suggested that for optimal estimation of ammonia derived flow parameters, there may be circumstances in which the ROI should be placed so as to partially overlap the LV cavity. However to reduce errors due to time shifts, our data suggest that (for the water model) the opposite ROI strategy should be employed-avoiding the LV cavity. As suggested by Hutchins [17], such opposing strategies can be reconciled in order to find an optimum ROI strategy only by a detailed examination of the uses to which the data will be put, and the kind of analysis to be performed.

The results obtained here agree with the findings in a previous report [11], in which longer total scanning times were also found to reduce the sensitivity of flow to time delay (Fig. 3). Our results, however, differ from this previous study in that we find smaller systematic errors. For example, for comparable parameters, $\Delta t_{1}=1 \mathrm{~s} ; \Delta t_{2}=0.8 \mathrm{~s} ; T_{t s}=1.5$ $\min ; P=0.5 ; f=1 \mathrm{ml} / \mathrm{g} / \mathrm{min} ; S=0.3$ (approximately the values in [11]), our systematic error in $\hat{f}$ is $18 \%$ while the result in [11] was close to $30 \%$. For regular duration scans we found a bias of $8 \%$ for $T_{t s}=4.25 \mathrm{~min}$ as against $10.5 \%$ for $T_{t s}=5.5 \mathrm{~min}$ in that reference. A possible explanation for this difference might be that the measurements in [11] were performed on a scanner with very low dead time and randoms. Our simulations show that the equivalent errors on the "ideal scanner," would be 27 and $12.4 \%$ - reasonably close to the values shown in [11].

\section{A. Correcting Time Delay Effects}

Obviously, in certain circumstances, a correction for time delay is required, particularly when short scans (which our data show are best avoided) are performed on ischemic patients and when $A(t)$ cannot be measured from the LV cavity. The results presented here suggest three approaches to deal with those situations in which the bias in $\hat{f}$ is significant

1) Use the four-parameter model as defined in $\mathrm{C}_{\mathrm{SC}}$. The $\delta t$ can be set to any value between 0 and $0.5 \mathrm{~s}$ without significantly influencing the bias of the fit. The increase of SD in this procedure is small and the bias produced by ignoring time delay is reduced or removed completely.
Even in the most extreme case simulated here $\left(T_{t s}=75\right.$ $\mathrm{s}$ and $f=0.2 \mathrm{ml} / \mathrm{g} / \mathrm{min}$ where the bias was $235 \%$ ), the use of a four-parameter model, defined in $\mathrm{C}_{\mathrm{SC}}$, reduces the flow bias to only $-0.4 \% \pm 2 \%$. Usually, the introduction of a fourth parameter greatly increases the variance in the measured flow value. For cardiac blood flow measurements, however, this increase in variance does not occur in the $\mathrm{C}_{\mathrm{SC}}$ model. Here the time delay can be estimated well, due to the sharply peaked appearance of the spill-over term which dominates the early data. This is also the reason why biases are large when no time shift correction is applied.

2) Measure two arterial input functions instead of a single one. The first would be measured at a location that provides a good estimate of $A(t)$ (e.g., in the left atrium, since the effects of myocardial counts in the ROI are minimized). This first arterial input function would be used in the convolution term of (2). The second measurement of $A(t)$ would be made inside the LV cavity, and this $A(t)$ would be used in the spill-over term of (2). In this way the spill-over term has no time delay $\left(\Delta t_{2}=0\right)$, and since the time discrepancy of $A(t)$ used in the convolution term does not produce significant bias, the three parameter fit would suffice. Although the data presented here suggest that this scheme should in principle work, we have not tested this approach in practice.

3) Artificially decrease the weights of early data, when performing fits. In this approach, although sensitivity to time delay would indeed be reduced, so too would the precision of the fit. In addition, as the weighting of early points was reduced, the precision of the spillover term would also be reduced. Such a scheme could, however, greatly reduce the sensitivity of flow to time shifts, especially for scanners with good count rate performance. The practical advantages and disadvantages of this method remain to be investigated.

There are two caveats that the reader should consider. First, with the exception of 1) above, the above suggested methods for potentially reducing the sensitivity to time shifts have not been exhaustively tested, and so their use in clinical practice remains speculative. Second, there are many other important factors, aside from the time delay effect investigated here, that can influence the accuracy and precision of the bolus method. Deadtime and randoms (as well as dosimetry) usually limit the injected dose which can be given, thereby limiting counting statistics, especially at low flows. The model is only valid when flow throughout the ROI is homogeneous-a condition that is rarely met in practice. In addition, the myocardial time activity curve can be contaminated with counts from the RV cavity (for septal regions) or with counts from the liver. The effect of such contamination has not been thoroughly studied, but it clearly will adversely affect the measurement of flow. All of the data presented in this paper have focused on one particular aspect of bolus water studies - the influence of time delay on flow. However, these other important factors must also be kept in mind when selecting an optimal experimental protocol for measuring blood flow. 


\section{CONCLUSION}

We have studied how time delays in the input function affect cardiac flow, when a bolus ${ }^{15} \mathrm{O}$ water flow model is used. Our results indicate that under many circumstances the estimates of flow are not very sensitive to the discrepancy in time between the arterial input curve $A(t)$ and the myocardial tissue time activity curve $M(t)$. However, we also find that if the total imaging time is excessively short (less than $2 \mathrm{~min}$ ) small time delays can produce large errors in flow. In addition, the bias in flow can become significant when the spill-over fraction is large (greater than 30\%), regardless of imaging time. We have proposed several schemes which might be employed to reduce the bias due to time delay in these circumstances. We conclude that in most experimental situations the bias in flow caused by time delays can be reduced to acceptable levels.

\section{REFERENCES}

[1] L. I. Araujo, A. A. Lammertsma, C. Rhodes, E. McFalls, H. Iida, E. Rechavia, A. Galassi, R. De Silva, T. Jones, and A. Maseri, "Noninvasive quantitation of regional myocardial blood flow in coronary artery disease with oxygen-15-labeled carbon dioxide inhalation and positron emission tomography," Circ., vol. 83, pp. 875-885, 1991.

[2] H. Iida, A. Takahashi, Y. Tamura, Y. Ono, and A. A. Lammertsma, "Myocardial blood flow: Comparison of oxygen-15-water bolus injection, slow infusion and oxygen-15-carbon dioxide slow inhalation," $J$. Nucl. Med., vol. 36, pp. 78-85, 1995.

[3] G. Wisenberg, H. R. Schelbert, E. J. Hoffman, M. E. Phelps, G. D. Robinson, C. E. Selin, J. Child, D. Skorton, and D. E. Kuhl, "In vivo quantitation of regional myocardial blood flow by positron-emission computed tomography," Circ., vol. 63, pp. 1248-1258, 1981.

[4] E. Henze, S. C. Huang, O. Ratib, E. Hoffman, M. E. Phelps, and H R. Schelbert, "Measurement of regional tissue and blood radiotracer concentrations from serial tomographic images of the heart," J. Nucl. Med., vol. 24, pp. 987-996, 1983.

[5] S. R. Bergmann, K. A. Fox, A. L. Rand, K. D. McElvany, M. J. Welch, J. Markham, and B. E. Sobel, "Quantification of regional myocardial blood flow in vivo with $\mathrm{H}_{2}^{15} \mathrm{O}$," Circ., vol. 70, pp. 724-733, 1984.

[6] S. C. Huang, M. Schwaiger, R. E. Carson, J. Carson, H. Hansen, C. Selin, E. J. Hoffman, N. MacDonald, H. R. Schelbert, and M. E. Phelps,
"Quantitative measurement of myocardial blood flow with oxygen-15 water positron emission tomography: An assessment of potential and problems," J. Nucl. Med., vol. 26, pp. 616-625, 1985.

[7] R. M. Knabb, K. A. Fox, B. E. Sobel, and S. R. Bergmann, "Characterization of the functional significance of subcritical coronary stenoses with $\mathrm{H}_{2}^{15} \mathrm{O}$ and positron-emission tomography," Circ., vol. 71, pp. 1271-1287, 1985.

[8] H. Iida, I. Kanno, A. Takahashi, S. Miura, M. Murakami, K. Takahashi, Y. Ono, F. Shishido, A. Inugami, N. Tomura, S. Higano, H. Fujita, H. Sasaki, H. Nakamichi, S. Mizusawa, Y. Kondo, and K. Uemura, "Measurement of absolute myocardial blood flow with $\mathrm{H}_{2}^{15} \mathrm{O}$ and dynamic positron-emission tomography," Circ., vol. 78, pp. 104-115, 1988.

[9] S. R. Bergmann, P. Herrero, J. Markham, C. J. Weinheimer, and M. N. Walsh, "Non-invasive quantitation of myocardial blood flow in human subjects with oxygen-15-labeled water and positron emission tomography," J. Amer. Coll. Cardiol., vol. 14, pp. 639-652, 1989.

[10] P. Herrero, J. Markham, and S. R. Bergmann, "Quantitation of myocardial blood flow with $\mathrm{H}_{2}^{15} \mathrm{O}$ and positron-emission tomography: Assessment and error analysis of a mathematical approach," J. Comput. Assist. Tomogr., vol. 13, pp. 862-873, 1989.

[11] P. Herrero, J. J. Hartman, M. J. Senneff, and S. R. Bergmann, "Effects of time discrepancies between input and myocardial time-activity curves on estimates of regional myocardial perfusion with PET," J. Nucl. Med., vol. 35, pp. 558-566, 1994.

[12] S. S. Kety, "The theory and applications of the exchange inert gas at the lung and tissues," Pharmacol. Rev., vol. 3, pp. 1-41, 1951.

[13] "Measurement of local blood flow by the exchange of an inert, diffusible substance," Methods. Med. Res., vol. 8, pp. 228-236, 1960.

[14] H. Iida, S. Higano, N. Tomura, F. Shishido, I. Kanno, S. Miura, M. Murakami, K. Takahashi, H. Sasaki, and K. Uemura, "Evaluation of regional differences of tracer appearance time in cerebral tissues using $\left[{ }^{15} \mathrm{O}\right]$ water and dynamic positron emission tomography," J. Cereb. Blood Flow, vol. 8, pp. 285-288, 1988.

[15] S. Pajevic, S. L. Bacharach, R. E. Carson, and V. Dilsizian, "Correction for time shifts in the cardiac $\mathrm{H}_{2}^{15} \mathrm{O}$ bloodflow model," J. Nucl. Med., vol. 35 , no. 5 , p. $183,1994$.

[16] H. Iida, C. Rhodes, R. De Silva, L. I. Araujo, P. Bloomfield, A Lammertsma, and T. Jones, "Use of the left ventricular time-activity curve as a noninvasive input function in dynamic oxygen-15-water positron emission tomography," J. Nucl. Med., vol. 33, pp. 1669-1677, 1992.

[17] G. D. Hutchins, J. M. Caraher, and R. R. Raylman, "A region of interest strategy for minimizing resolution distortions in quantitative myocardial PET studies," J. Nucl. Med., vol. 33, pp. 1243-1250, 1992. 